
\title{
$\angle$ Research Square \\ FOXA3: A Novel Tumor Suppressor in Esophageal Squamous Cell Carcinoma
}

\section{Siang Zhang}

Shanghai Changzheng Hospital https://orcid.org/0000-0001-6418-9314

\section{Yuxiang Jin}

Changzheng Hospital: Shanghai Changzheng Hospital

\section{Qianyu Han}

Changzheng Hospital: Shanghai Changzheng Hospital

\section{Xuewei Zhao}

Shanghai Changzheng Hospital

Lei Xue ( $\boldsymbol{\nabla}$ tommyxuel@smmu.edu.cn )

Shanghai Changzheng Hospital

\section{Research}

Keywords: FOXA3, esophageal squamous cell carcinoma, tumor suppressor, biomarker

Posted Date: October 1st, 2021

DOI: https://doi.org/10.21203/rs.3.rs-889419/v1

License: (c) (1) This work is licensed under a Creative Commons Attribution 4.0 International License. Read Full License 


\section{Abstract}

Background: Forkhead box A(FOXA) family, have been widely investigated in cancer research. However, currently the study of FOXA3 in malignant tumors is still limited compared with other family members. FOXA1 and FOXA2 both play a role in the development and progression of esophageal cancer and Barrett's esophagus, but the exact role of FOXA3 in esophageal squamous cell carcinoma (ESCC) has not been elucidated.

Methods: The expression of FOXA3 in ESCC tissues was determined by real-time PCR immunohistochemistry staining. The relationship between FOXA3 and overall survival rate of patients was analyzed by Kaplan-Meier analysis, and Cox regression analysis was used to analyze independent prognostic risk factors. Cell proliferation was analyzed by CCK-8 assay, EdU assay and colony forming assay. Cell migration was analyzed by wound healing assay and Transwell assay. Tumorigenesis by subcutaneous injection was performed to detect the inhibition of tumors by FOXA3 in vivo.

Results: We found that FOXA3 is down-regulated in ESCC tissues and low FOXA3 expression significantly correlated with a poor prognosis of ESCC. Additionally, after up-regulating the FOXA3 expression, the abilities of proliferation, migration and invasion in ESCC cells were significantly inhibited. Moreover, overexpression of FOXA3 can inhibit tumor growth in vivo either via tumorigenesis assay by subcutaneous injection.

Conclusions: Low FOXA3 expression significantly correlated with a poor prognosis of ESCC and the overexpression of FOXA3 could inhibit the growth and metastasis of ESCC, which indicated that FOXA3 maybe a novel biomarker and therapeutic target for ESCC.

\section{Background}

Esophageal cancer is a common lethal malignant tumor worldwide, and can be mainly divided into 2 histologic subtypes: esophageal squamous cell carcinoma(ESCC) and esophageal adenocarcinoma(EAC), with distinct geographical difference in the incidence[1]. The morbidity and mortality in China are higher than the world average level and the majority of them in China are ESCC[2].The symptoms of esophageal cancer in early stage are insidious, patients are often diagnosed until advanced stage, losing the opportunity for radical surgery. Beyond that, even after receiving surgical treatment and other traditional therapeutic methods, however, the overall treatment remains unsatisfactory $[3,4]$. The current treatment principles for ESCC and EAC are roughly the same, mainly depend on which stage tumor is in, but they are actually two different diseases since the much different oncology characteristics[5]. In the context of the rapid development of molecular targeted therapy in malignant tumor and the promising effect it brought, it is urgently required to clarify the molecular mechanisms of development and progression relevant to esophageal cancer, particularly ESCC.

The FOX family, known as forkhead box proteins, are the transcription factors characterized by a 110 amino acid length and highly conserved DNA binding domain, sharing homology in the Drosophila 
forkhead gene[6]. And they are further categorized as 19 subfamilies from FOXA to FOXS according to the sequence of the forkhead box. The Fox family subfamily A (FOXAs) consists of three isotypes: FOXA1/hepatocyte nuclear factor (HNF)3a, FOXA2/HNF3 $\beta$ and FOXA3/HNF3y. Since the 110 amino acid so called forkhead box of FOXAs are structurally similar with the DNA binding motif of linker histones, which can affect chromatin structure directly. This property makes it known as "pioneer factors" that bind to promoters and enhancers enable chromatin access for other transcription factors to play a downstream role[7, 8]. The FOXA family are necessary for the organogenesis and differentiation such as liver, pancreas, lung and prostate. They can also regulate metabolism by regulating multiple target genes in liver, pancreas and adipose tissue to participate in the occurrence and development of disease [9-11]. In addition, the FOXA family is closely related to the incidence, progression and metastasis of malignant tumors[12-14]. Currently, FOXA1 and FOXA2 have been studied extensively, while the researches on FOXA3 have mainly focused on embryonic development, glucose and lipid metabolism and related diseases, as well as the newer field of liver regeneration and hepatic reprogramming $[15,16]$. Up to now, the research of FOXA3 in malignant tumors is still limited compared with other family members.

FOXA1 and FOXA2 both play a role in the development and progression of esophageal cancer and Barrett's esophagus[17-20]. And it is reported that FOXA3 is highly expressed in esophageal cancer and Barrett's esophagus, and the invasion and migratory ability of EC cells following FOXA3 knockdown was significantly reduced.Furthermore, FOXA1, FOXA2 and FOXA3 were found to play synergistic roles in metastasis of esophageal cancer[21].

However, in our preliminary experiment, we found FOXA3 expression was down-regulated in human ESCC tissues by 11 fresh specimens. Hence we hypothesized that FOXA3 may play a different role as previously reported[21]. In this study, we examined the expression of FOXA3 in ESCC patients, analyze its correlation with prognosis and subsequently utilized a series assays to figure out the exact role of FOXA3 in ESCC.

\section{Methods}

\section{Patients and tissue samples}

All cases of tissue samples were collected from ESCC patients underwent esophagectomy and following two or three-field lymph node dissection at the Department of Thoracic Surgery of Changzheng Hospital, Naval Military Medical University. Of which 93 patients underwent surgical resection between January 2009 to December 2010, and regarding clinicopathological characteristics of the patients are presented in table 1. Informed consent was obtained from

each patient and this study was approved by the Ethics Committee of Changzheng Hospital.

\section{Cell lines}


ESCC cell lines TE-1 and Eca-109 were obtained from Shanghai Institute of Biochemistry and Cell Biology, Chinese Academy of Sciences. All cells were cultured in DMEM high-glycemic medium containing $10 \%$ fetal bovine serum at $37^{\circ} \mathrm{C}, 5 \% \mathrm{CO}_{2}$.

\section{Quantitative real-time PCR (qRT-PCR)}

Total RNAs were extracted from tissues and cells with TRI Reagent $\circledast($ Sigma). The reverse transcription and $q R T-P C R$ was performed using Bestar ${ }^{\circledR}$ one step $q R T-P C R$ kit (Sybr Green). Glyceraldehyde-3-

phosphate dehydrogenase(GAPDH) was used as an internal control. The primer sequences used in this paper were shown in Table 2. 
Table 1

Clinicopathologic characteristics of ESCC patients in relation to expression levels of FOXA3

\begin{tabular}{|c|c|c|c|}
\hline Clinicopathologic parameter & $\begin{array}{l}\text { FOXA3 Low } \\
(\mathrm{N}=46)\end{array}$ & $\begin{array}{l}\text { FOXA3 High } \\
(\mathrm{N}=47)\end{array}$ & P-value \\
\hline \multicolumn{4}{|l|}{ Age } \\
\hline$<65$ & 21 & 24 & \multirow[t]{2}{*}{0.753} \\
\hline$\geq 65$ & 25 & 23 & \\
\hline \multicolumn{4}{|l|}{ Gender } \\
\hline Male & 38 & 39 & \multirow[t]{2}{*}{1.000} \\
\hline Female & 8 & 8 & \\
\hline \multicolumn{4}{|l|}{ Size of tumor $(\mathrm{cm})$} \\
\hline$<5$ & 27 & 25 & \multirow[t]{2}{*}{0.745} \\
\hline$\geq 5$ & 19 & 22 & \\
\hline \multicolumn{4}{|l|}{ T-stage } \\
\hline $\mathrm{T} 1-\mathrm{T} 2$ & 12 & 11 & \multirow[t]{2}{*}{0.953} \\
\hline T3-T4 & 34 & 36 & \\
\hline \multicolumn{4}{|l|}{$\mathrm{N}$-stage } \\
\hline NO & 13 & 29 & \multirow[t]{2}{*}{0.002} \\
\hline N1-N3 & 33 & 18 & \\
\hline \multicolumn{4}{|l|}{ Differentiation } \\
\hline G1 & 7 & 7 & \multirow[t]{2}{*}{1.000} \\
\hline G2-G3 & 39 & 40 & \\
\hline \multicolumn{4}{|l|}{ p-TNM stage } \\
\hline $\mathbb{Q}-\mathbb{Q}$ & 16 & 28 & \multirow[t]{2}{*}{0.029} \\
\hline $\mathbb{Q}-\mathbb{Z}$ & 30 & 19 & \\
\hline
\end{tabular}

\section{Immunohistochemistry(IHC) staining}

ESCC tissue slides were deparaffinized and rehydrated, heat-induced antigen retrieval and blocked with $5 \%$ BSA. Thereafter, the tissue samples were incubated overnight at $4^{\circ} \mathrm{C}$ with FOXA3 primary antibody (Sigma,rabbit, 1:1000) followed by incubation with secondary antibody for $30 \mathrm{~min}$. The IHC staining were 
evaluated independently by three pathologists, and the grade of FOXA3 expression were scored according to the intensity of staining and the percentage of positive cells ( 0 : no staining; $1: \leq 10 \% ; 2: 10-50 \% ; 3$ : $\geq 50 \%$ ). According to scores averaged, the expression of FOXA3 was divided into two groups : high expression group $(2,3)$ and low expression group $(0,1)$.

\section{Cell transfections}

Recombinant lentiviral vectors was obtained from HanBio Technology Co.Ltd. (Shanghai, China). The resultant plasmid was sequenced to confirm the authenticity of the insert. Eca-109 and TE-1 cells were transfected with fresh lentiviral vectors expressing either vector control (LV-Con) or LV-FOXA3 construct in medium for 24 hours. Stable cells lines were generated by selecting transfected cells in cultures containing $2 \mu \mathrm{g} / \mathrm{ml}$ puromycin (HanBio, China) for 3 days. FOXA3 expression level in LV-FOXA3 and LVCon construct was then analyzed by qRT-PCR and Western blot.

\section{Western-Blot assay}

RIPA lysis buffer was used to extract protein from cell lysis and equal amounts of proteins were separated by SDS-PAGE and transferred to nitrocellulose filter membranes. Then the membranes were blocked with $5 \%$ skimmed milk for 1 hour and incubated with primary antibody against FOXA3(ab108454, 1:500, Santa Cruz Biotechnology) or GAPDH(1:5000, Santa Cruz Biotechnology) at $4^{\circ} \mathrm{C}$ overnight. After washed 3 times, the membranes were incubated with secondary antibodies for 1 hour. All western blots were visualized with the enhanced chemiluminescence substrate.

\section{Colony forming assay}

Each group of cells was seeded on petri dishes and maintained for 2 weeks, respectively. Then $4 \%$ paraformaldehyde and crystal violet were used to fix and stain the colonies and visible colonies were counted manually after taking photographs.

\section{CCK-8 assay}

Cells were seeded on 96 -well plates (1000/well) and incubated at $37^{\circ} \mathrm{C}, 5 \% \mathrm{CO} 2$ for 24,48 and 72 hours. Then cell counting kit8 (R\&D Systems) was used at each well according to the manufacturer's protocol for the cell proliferation assay. Cell counts were measured in triplicate using the microplate readers(BioTek Instruments) at $450 \mathrm{~nm}$. And then the cell proliferation curve was plotted according to the $\mathrm{OD}$ value.

\section{5-Ethynyl-2'-deoxyuridine (EdU) assay}

Cell proliferation was measured using the EdU assay kit(RiboBio, China). Firstly cells were seeded into 96well plates and incubated incubated for 2 hours at $37^{\circ} \mathrm{C}$ after the addition of EdU $(50 \mu \mathrm{M})$. According to the manufacturer's protocol, describe it briefly, cells were then fixed in $4 \%$ paraformaldehyde for 30 minutes and permeabilized with $0.5 \%$ TritonX-100 for 10 minutes at room temperature. After washed with PBS, $1 \times$ Apollo $\circledast$ staining reaction $(100 \mu \mathrm{l})$ was added to react with the EdU in the dark for 30 minutes. Subsequently, Hoechest33342 (100 $\mu$ l) was added for 30 minutes to visualize cell nucleus. Images of 
cells were obtained under a fluorescence microscope (OLYMPUS, Japan). Cell proliferation was analyzed based on the percentage of EdU-positive cells for each sample.

\section{Wound healing assay}

Cells were seeded on 6 -well dish at a density of $3 \times 10^{6} / \mathrm{ml}$ and grown to $90 \%$ confluence. A $200 \mu$ pipette tip was applied for making a straight line in each well, and images were captured at the 0 and 48 hour in microscope.

\section{Transwell assay}

Tumor cell migration assays were performed using a Transwell chamber with $8 \mu \mathrm{m}$ pores in 24-well dishes. The cells $\left(2 \times 10^{5} / \mathrm{ml}\right)$ were suspended in serum-free medium and seeded in upper chambers, and lower chambers were supplemented with medium containing 10\% FBS. After incubation for 24 hours, cells in the upper chamber were washed out and the cells that had migrated into the membrane were fixed and stained with crystal violet. Cellular quantification was based on the average of cell number counting in five random fields under microscope ( $\times 100$ magnification).

As the difference, cell invasion assays were investigated using a Transwell system coated with matrigel $(8 \mu \mathrm{m}, \mathrm{BD})$. The rest of the detailed steps are the same as above.

\section{Tumorigenesis by subcutaneous injection}

All animal experiments were approved by the Medical Experimental Animal Care Commission in Navel Military Medical University. Mice at age of 6 weeks were randomly divided into two groups, Eca-109 (LVCon) and Eca-109 (LV-FOXA3) were injected subcutaneously into the back of nude mice $\left(2 \times 10^{6} / \mathrm{ml}\right)$. Six weeks after the injection, the mice were sacrificed and the tumors were removed to measure the weight.

\section{Statistical analysis}

Data are expressed as the mean \pm standard error of the mean (SEM). Categorical variables and continuous variables were analyzed by Chi-squared test and Mann-Whitney $\mathrm{U}$ test, respectively. Univariate and multivariate analysis for overall survival (OS) were performed by Kaplan-Meier survival analysis and Cox regression analysis. OS was described as the time frame between date of surgery to the date of death or the last follow up date: July 2015. Statistical significance level was $p$-values $<0.05$. Sometimes, $p<0.01$ were used to show extreme significance. Statistical analyses were achieved using the SPSS version 22.0

\section{Results}

\section{FOXA3 is down-regulated in human ESCC tissues}

Firstly we collected 11 human ESCC tissue samples and paired adjacent normal tissues to detect the expression of FOXA3 by RT-PCR. As shown in Fig. 1A, we found FOXA3 expression were lower in human 
ESCC tissues compared with the paired adjacent normal tissues $(p=0.022)$. To determine whether FOXA3 was down-regulated in ESCC tissues, we further performed IHC staining analysis to examine the expression level of FOXA3 in 93 paraffin-embedded, archived ESCC tissues and paired adjacent normal tissues. The staining intensity of FOXA3 in 68/93 of normal tissues was noticeably stronger than that in tumors, and the representative image is shown in Fig. 1B, suggesting that FOXA3 may play an important role in ESCC.

\section{Association of FOXA3 expression with clinicopathological characteristics}

To further explore the role of FOXA3 in the development and progression of ESCC, we analyzed the correlation between FOXA3 expression levels and the clinicopathological characteristics of ESCC patients (age, gender,tumor size, histology grade, TMN stage, T grade and lymph node metastasis status). All of ESCC patients were divided into two groups according to FOXA3 expression level. As shown in Table 1, FOXA3 expression levels correlated negatively with the lymph nodes metastasis $(P=0.002)$, and has no significant correlation with the patients' gender, age, tumor size and differentiation. In addition, FOXA3 was also found to be correlated with the pTNM stage $(p<0.05)$. However, considering that there were only 5 cases of stage I and stage IV among 93 cases and the main difference between stage II and stage III cases is whether there is lymph node metastasis(N0 or N1-3) which is the main factor causing the correlation between FOXA3 and PTNM stage. Subsequent studies need include more early and advanced stage cases to further clarify the correlation.

Based on Kaplan-Meier survival analysis, higher FOXA3 expression predicted longer survival of ESCC patients compared with low expression group. $(p=0.001$, Fig. 2$)$. In univariate Cox regression analysis, lymph node metastasis and low FOXA3 expression showed a signifcant association with poorer overall survival ( $p=0.001$ and $p=0.002$ respectively, Table 3 ). Multivariate Cox regression analysis also revealed that lymph node metastasis and FOXA3 expression were independent prognostic factors for overall survival in ESCC patients ( $p=0.044$ and $p=0.032$ respectively, Table 4).

Table 2

The primer sequences used for qRT-PCR

\begin{tabular}{|lll|}
\hline Gene & Forward Primer sequence $\left(5^{\prime} \mathbf{3}^{\prime} \mathbf{)}\right.$ & Reverse Primer sequence $\left(\mathbf{5}^{\prime} \mathbf{-} \mathbf{3}^{\prime} \mathbf{)}\right.$ \\
\hline FOXA3 & CTGGCCGAGTGGAGCTACTA & AGGGGGATAGGGAGAGCTTA \\
\hline B-actin & AATCGTGCGTGACATTAAGGAG & ACTGTGTTGGCGTACAGGTCTT \\
\hline
\end{tabular}


Table 3

Overall survival of ESCC patients: univariate analysis

\begin{tabular}{|llll|}
\hline Clinicopathologic parameter & HR & $\mathbf{9 5 \%} \mathbf{C l}$ & P-value \\
\hline Gender & 1.055 & $0.548-2.030$ & 0.872 \\
\hline Age & 1.309 & $0.786-2.178$ & 0.302 \\
\hline Size of tumor & 0.693 & $0.411-1.168$ & 0.169 \\
\hline T-stage & 0.965 & $0.538-1.732$ & 0.906 \\
\hline Lymph node metastasis & 2.327 & $1.370-3.953$ & 0.002 \\
\hline Differentiation & 1.234 & $0.586-2.601$ & 0.580 \\
\hline FOXA3 & 0.422 & $0.251-0.709$ & 0.001 \\
\hline
\end{tabular}

Table 4. Overall survival of ESCC patients: multivariate analysis

\begin{tabular}{|llll|}
\hline Clinicopathologic parameter & HR & $\mathbf{9 5 \%} \mathbf{C l}$ & P-value \\
\hline Lymph node metastasis & 1.811 & $1.016-3.228$ & 0.044 \\
\hline FOXA3 & 0.539 & $0.306-0.948$ & 0.032 \\
\hline
\end{tabular}

FOXA3 inhibits the proliferation of ESCC cells.

After transfected cells were stably generated, the expression level of FOXA3 was determined via RT-qPCR and Western Blot. As shown in Fig. 3A, the mRNA levels of FOXA3 were significantly up-regulated. The result was also verified by Western-Blot, compared with the control group, FOXA3 levels were remarkably increased in LV-FOXA3 transfected ESCC cells(Fig. 3B).

The influence of FOXA3 on ESCC cell proliferation was then investigated using the CCK-8 assay. It was shown that the proliferation rate of ESCC cells in the LV-FOXA3 was significantly decreased relative to that of the control group(Fig. 4A). The EdU assay was also performed as a more sensitive and specific method to evaluate the effect of FOXA3 on proliferation. As shown in Fig. 4C, the number of ESCC cells binding EdU in the LV-FOXA3 group was distinctly decreased compared with the number in the control group. In addition, the long-term influence of FOXA3 on cell proliferation was assessed using the colony formation assay. The results revealed that FOXA3 overexpression impaired colony formation ability (Fig. 4B). These observations indicated that FOXA3 overexpression can inhibit the proliferation of ESCC cells.

\section{FOXA3 inhibits the tumorigenicity in vivo}

To investigate the effects of FOXA3 on tumorigenicity in vivo, Eca-109 cells transfected with the vectors mentioned above were injected into the back of nude mice to generate tumors ectopically. As shown in 
Fig. 5, the average tumor weight of the FOXA3 overexpression group (LV-FOXA3) was $0.6586 \pm 0.1764 \mathrm{~g}$, which was significantly lighter than the control group $2.021 \pm 0.1162 \mathrm{~g}(\mathrm{P}<0.05)$. This result indicated that FOXA3 can also inhibit tumor growth in vivo.

\section{FOXA3 inhibits migration and invasion of ESCC cells}

Since it has been found that lower expression of FOXA3 is closely related to lymph node metastasis, we speculate that FOXA3 can not only inhibit tumor proliferation, but also play a critical role in tumor

metastasis. Tumor metastasis itself is the greatest cause of death in cancer patients, and is an extremely complex, multi-step process that involves the migration and invasion of cancer cells, which is the key step in cancer metastasis. Thus we evaluated the ability of cell migration and invasion in Eca-109 and TE-1 cells line. As as shown in Fig. 6, compared to the control groups, the migration of Eca-109 and TE-1 cells were both decelerated significantly when FOXA3 were up-regulated. The results of Transwell migration assay showed that the number of cells migrating in the LV-FOXA3 groups was apparently fewer compared with the control groups (Fig. 7A). Likewise, compared with control groups, both Eca-109 and TE-1 cells exhibited significantly decreased invasive tendencies after FOXA3 were up-regulated(Fig. 7B). These results were in accordance with our clinical data, indicating the suppressive role of FOXA3 in the metastasis of ESSC.

\section{Discussion}

ESCC is the seventh most common cancer and the sixth leading cause of cancer death globally[1]. Despite advances in the diagnosis and surgical treatment of the disease during the past several decades, the prognosis for patients remains disappointing. Therefore, finding new molecular biological markers and effective therapeutic targets for ESCC are urgently required.

According to previous studies, All FOXA members play a key role in esophagus cancer, and the FOXA3's effect on esophagus cancer seems to be inconsistent with the results of our preliminary experiments which the expression of FOXA3 mRNA is down-regulated in human ESCC tissues ( $n=11)$ by using RTPCR.

In this study, the expression level of FOXA3 in ESCC tissues ( $n=93)$ were examined using immunohistochemistry. Subsequently, we investigated the correlation of FOXA3 with clinicopathologic characteristics of ESCC patients. Kaplan-Meier survival analysis indicated that ESCC patients with low FOXA3 expression in general had worse prognosis than those with high FOXA3 expression. Univariate and multivariate Cox regression analysis indicated that low FOXA3 expression was an independent risk factor for the prognosis of ESCC patients. The clinical data also showed that low level of FOXA3 was correlated with lymph node metastasis.

To further explore whether FOXA3 is involved in the development of ESCC as a tumor suppressor factor, we used CCK8 assay, colony formation assay and EdU assay to test the proliferation ability of ESCC, and the results all confirmed that FOXA3 overexpression could indeed inhibit the proliferation of ESCC cells 
effectively. Subsequently, the Eca-109 cells were injected subcutaneously into nude mice. And it was shown that the average weight of implanted tumor in FOXA3 overexpression group is significantly lighter than control group which proved that FOXA3 could also inhibit tumor growth in vivo.

Since the 93 cases we chosen were all post-operation patients, there was no distant metastasis before surgery, we still found that lower expression of FOXA3 was closely related to lymph node metastasis, thus we speculate that FOXA3 can not only inhibit tumor proliferation, but also affect tumor metastasis. Tumor metastasis itself is the biggest cause of death in cancer patients and is an extremely complex process involving multiple steps[22, 23]. Furthermore, the ability of cell invasion and migration is considered the most crucial to tumor metastasis[24]. The results of wound healing assay and Transwell migration/invasion assay indicated that the ability of invasion and migration in ESCC cell was either significantly reduced after overexpression of FOXA3.

Taking these results together, FOXA3 may exert tumor suppressive roles in ESCC via the inhibition of cell proliferation and invasion activities. However, intriguingly, it is previously reported FOXA3 is highly expressed in esophageal cancer and is closely related to the metastasis of esophageal cancer[21]. To analyze that, it's worth noting that the esophageal cancer case data in the GSE6059, GSE13898, and Oncomine datasets chosen in the above study did not distinguish the specific pathological type of esophageal cancer, and 96 pairs of surgically removed tumor tissues also covered multiple pathological types involving not only ESCC, but also undifferentiated carcinoma, and Siewert type I gastroesophageal junction adenocarcinoma. Furthermore, the specific number of each pathological type was not mentioned. Since ESCC and EAC are obviously two different diseases according to their oncogenic characteristics and all tissue specimens we chose for our study are all ESCC, it is understandable that the two studies have inconsistent results.

The limitation of this study is that there was only subcutaneous tumorigenesis assay was done on nude mice and the effect of FOXA3 on tumor metastasis in vivo have not been studied yet. The lack of research on the mechanism of how FOXA3 is involved in the process of tumor proliferation and metastasis is another disadvantage which may also partly explain the inconsistency with the results of previous studies.

The specific role of FOXA3 maybe controversial, but there is no doubt that FOXA3 is quite crucial for the development and progression of ESCC, and FOXA3 is expected to become a molecular biomarker, a prognostic indicator and an effective therapeutic target for ESCC. Therefore, in the next step, animal models of tumor metastasis need to be constructed to clarify the role of FOXA3 on metastasis in vivo, and further research should be carried out to seek the signaling pathways and molecular networks involved in the function of FOXA3 in ESCC.

\section{Conclusion}

In summary, we have reached the preliminary conclusion that low FOXA3 expression significantly correlated with a poor prognosis of ESCC and FOXA3 might play a suppressive role in the growth and 
metastasis of ESCC. These results may provide a novel potential target for the treatment of ESCC.

\section{Abbreviations}

\begin{tabular}{|ll|}
\hline ESCC & Esophageal squamous cell carcinoma \\
\hline EAC & Esophageal adenocarcinoma \\
\hline FOXA3 & Forkhead box A3 \\
\hline qRT-PCR & Quantitative real-time polymerase chain reaction \\
\hline IHC staining & Immunohistochemistry staining \\
\hline
\end{tabular}

\section{Declarations}

\section{Ethics approval and consent to participate}

The experimental protocol was established, according to the ethical guidelines of the Helsinki Declaration and was approved by the Ethics Committee of Changzheng Hospital. Informed consent was obtained from each patient.

\section{Consent for publication}

Not applicable

\section{Availability of data and materials}

Not applicable

\section{Competing interests}

The authors declare that they have no competing interests.

\section{Funding}

Not applicable

\section{Authors' contributions}

SZ completed the study of FOXA3 expression levels in ESCC samples, completed the conceptualization of the next experiments and was a major contributor in writing the manuscript. YJ performed a series of proliferation and migration assays of ESCC cell lines, and was responsible for the refinement of the first draft. QH completed the part of the animal experiment and completed most of the data analysis. All authors read and approved the final manuscript. 
Acknowledgements

Not applicable

\section{References}

1. Bray F, Ferlay J, Soerjomataram I, Siegel RL, Torre LA, Jemal A. Global cancer statistics 2018: GLOBOCAN estimates of incidence and mortality worldwide for 36 cancers in 185 countries. CA Cancer J Clin. 2018;68:394-424.

2. Zhou M, Wang H, Zeng X, Yin P, Zhu J, Chen W, Li X, Wang L, Wang L, Liu Y, Liu J, Zhang M, Qi J, Yu S, Afshin A, Gakidou E, Glenn S, Krish VS, Miller-Petrie MK, Mountjoy-Venning WC, Mullany EC, Redford SB, Liu H, Naghavi M, Hay SI, Wang L, Murray C, Liang X Mortality, morbidity, and risk factors in China and its provinces, 1990-2017: a systematic analysis for the Global Burden of Disease Study 2017. LANCET 2019, 394: 1145-1158.

3. Enzinger PC, Mayer RJ. Esophageal cancer. N Engl J Med. 2003;349:2241-52.

4. Dubecz A, Gall I, Solymosi N, Schweigert M, Peters JH, Feith M, Stein HJ. Temporal trends in longterm survival and cure rates in esophageal cancer: a SEER database analysis. J THORAC ONCOL. 2012;7:443-7.

5. Testa U, Castelli G, Pelosi E. Esophageal Cancer: Genomic and Molecular Characterization, Stem Cell Compartment and Clonal Evolution. Medicines (Basel) 2017, 4.

6. Kaestner KH, Knochel W, Martinez DE. Unified nomenclature for the winged helix/forkhead transcription factors. Genes Dev. 2000;14:142-6.

7. Cirillo LA, Lin FR, Cuesta I, Friedman D, Jarnik M, Zaret KS. Opening of compacted chromatin by early developmental transcription factors HNF3 (FoxA) and GATA-4. MOL CELL. 2002;9:279-89.

8. Sekiya T, Muthurajan UM, Luger K, Tulin AV, Zaret KS. Nucleosome-binding affinity as a primary determinant of the nuclear mobility of the pioneer transcription factor FoxA. Genes Dev. 2009;23:804-9.

9. Friedman JR, Kaestner KH. The Foxa family of transcription factors in development and metabolism. CELL MOL LIFE SCI. 2006;63:2317-28.

10. Xu L, Panel V, Ma X, Du C, Hugendubler L, Gavrilova O, Liu A, McLaughlin T, Kaestner KH, Mueller E. The winged helix transcription factor Foxa3 regulates adipocyte differentiation and depot-selective fat tissue expansion. MOL CELL BIOL. 2013;33:3392-9.

11. Zhao Y, Li HX, Wang K, Yan BY, Li W. Regulation of testicular steroidogenesis by Foxa3 via transcriptional modulation of ERalpha signaling in type 2 diabetes mellitus (T2DM). Biochem Biophys Res Commun. 2017;490:786-93.

12. Huang $\mathrm{C}$, Liu J, Xiong B, Yonemura Y, Yang X. Expression and prognosis analyses of forkhead box A (FOXA) family in human lung cancer. GENE. 2019;685:202-10. 
13. Le Gallo M, Rudd ML, Urick ME, Hansen NF, Merino MJ, Mutch DG, Goodfellow PJ, Mullikin JC, Bell DW. The FOXA2 transcription factor is frequently somatically mutated in uterine carcinosarcomas and carcinomas. CANCER-AM CANCER SOC. 2018;124:65-73.

14. Badve S, Turbin D, Thorat MA, Morimiya A, Nielsen TO, Perou CM, Dunn S, Huntsman DG, Nakshatri H. FOXA1 expression in breast cancer-correlation with luminal subtype $A$ and survival. CLIN CANCER RES. 2007;13:4415-21.

15. Cheng Z, He Z, Cai Y, Zhang C, Fu G, Li H, Sun W, Liu C, Cui X, Ning B, Xiang D, Zhou T, Li X, Xie W, Wang $H$, Ding J. Conversion of hepatoma cells to hepatocyte-like cells by defined hepatocyte nuclear factors. CELL RES. 2019;29:124-35.

16. Horisawa K, Udono M, Ueno K, Ohkawa Y, Nagasaki M, Sekiya S, Suzuki A. The Dynamics of Transcriptional Activation by Hepatic Reprogramming Factors. MOL CELL. 2020;79:660-76.

17. Lin L, Miller CT, Contreras JI, Prescott MS, Dagenais SL, Wu R, Yee J, Orringer MB, Misek DE, Hanash SM, Glover TW, Beer DG. The hepatocyte nuclear factor 3 alpha gene, HNF3alpha (FOXA1), on chromosome band $14 q 13$ is amplified and overexpressed in esophageal and lung adenocarcinomas. CANCER RES. 2002;62:5273-9.

18. Sano M, Aoyagi K, Takahashi H, Kawamura T, Mabuchi T, Igaki H, Tachimori Y, Kato H, Ochiai A, Honda H, Nimura Y, Nagino M, Yoshida T, Sasaki H. Forkhead box A1 transcriptional pathway in KRT7-expressing esophageal squamous cell carcinomas with extensive lymph node metastasis. INT J ONCOL. 2010;36:321-30.

19. Watts JA, Zhang C, Klein-Szanto AJ, Kormish JD, Fu J, Zhang MQ, Zaret KS. Study of FoxA pioneer factor at silent genes reveals Rfx-repressed enhancer at $\mathrm{Cdx} 2$ and a potential indicator of esophageal adenocarcinoma development. PLOS GENET. 2011;7:e1002277.

20. Wang DH, Tiwari A, Kim ME, Clemons NJ, Regmi NL, Hodges WA, Berman DM, Montgomery EA, Watkins DN, Zhang X, Zhang Q, Jie C, Spechler SJ, Souza RF. Hedgehog signaling regulates FOXA2 in esophageal embryogenesis and Barrett's metaplasia. J CLIN INVEST. 2014;124:3767-80.

21. Chen B, Yu J, Lu L, Dong F, Zhou F, Tao X, Sun E. Upregulated forkhead-box A3 elevates the expression of forkhead-box A1 and forkhead-box A2 to promote metastasis in esophageal cancer. ONCOL LETT. 2019;17:4351-60.

22. Steeg PS. Tumor metastasis: mechanistic insights and clinical challenges. NAT MED. 2006;12:895904.

23. Steeg PS. Targeting metastasis. NAT REV CANCER. 2016;16:201-18.

24. Friedl P, Mayor R. Tuning Collective Cell Migration by Cell-Cell Junction Regulation. CSH PERSPECT BIOL. 2017;9:a29199.

\section{Figures}


A
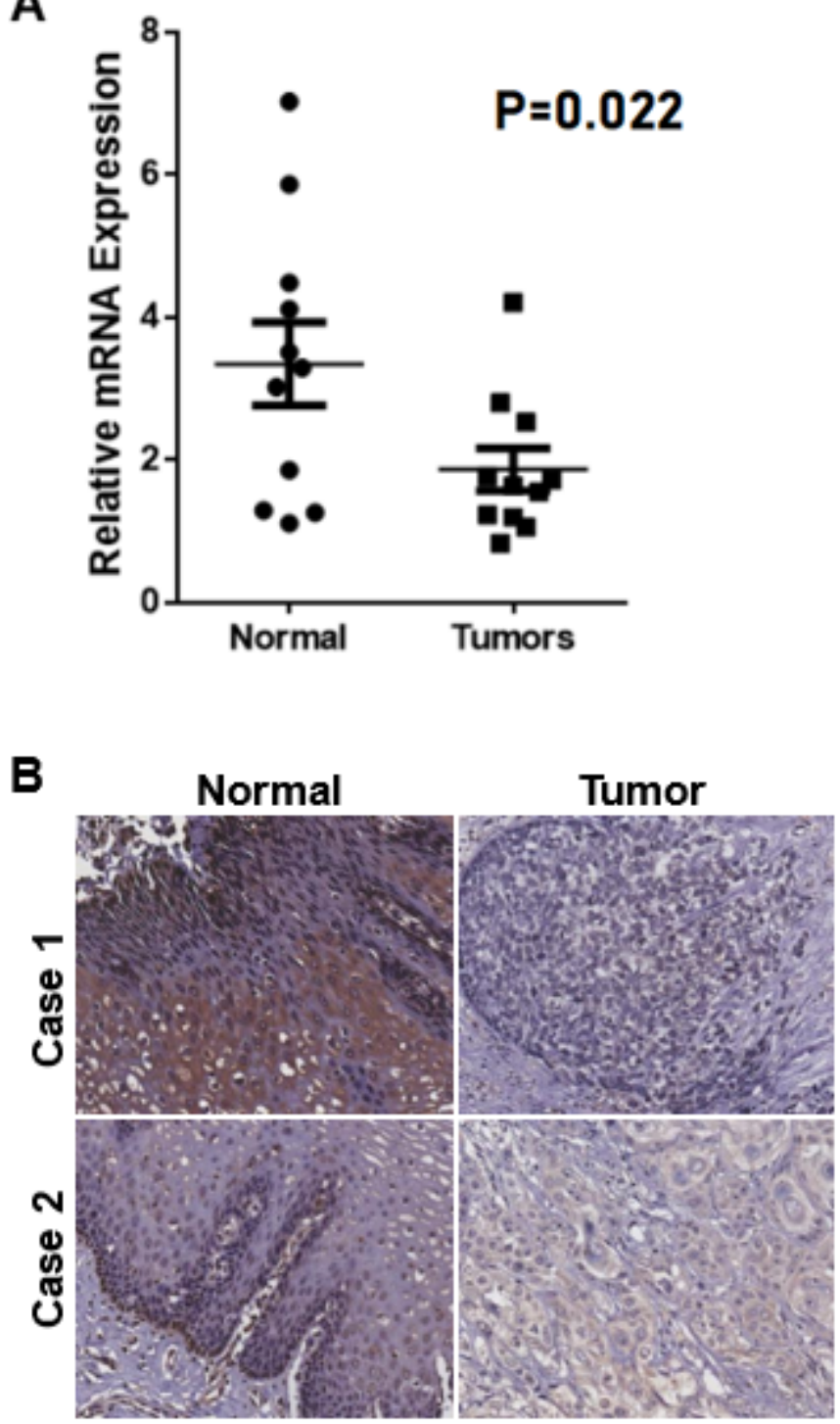

$(\times 400)$

Figure 1

FOXA3 is down-regulated in human ESCC tissues. A, The expression levels of FOXA3 in 11 pairs of human ESCC tissue samples and adjacent normal tissues were explored using RT-PCR. B, The representative images of expression levels of FOXA3 in ESCC and paired adjacent normal tissues from 93 patients by IHC staining. 


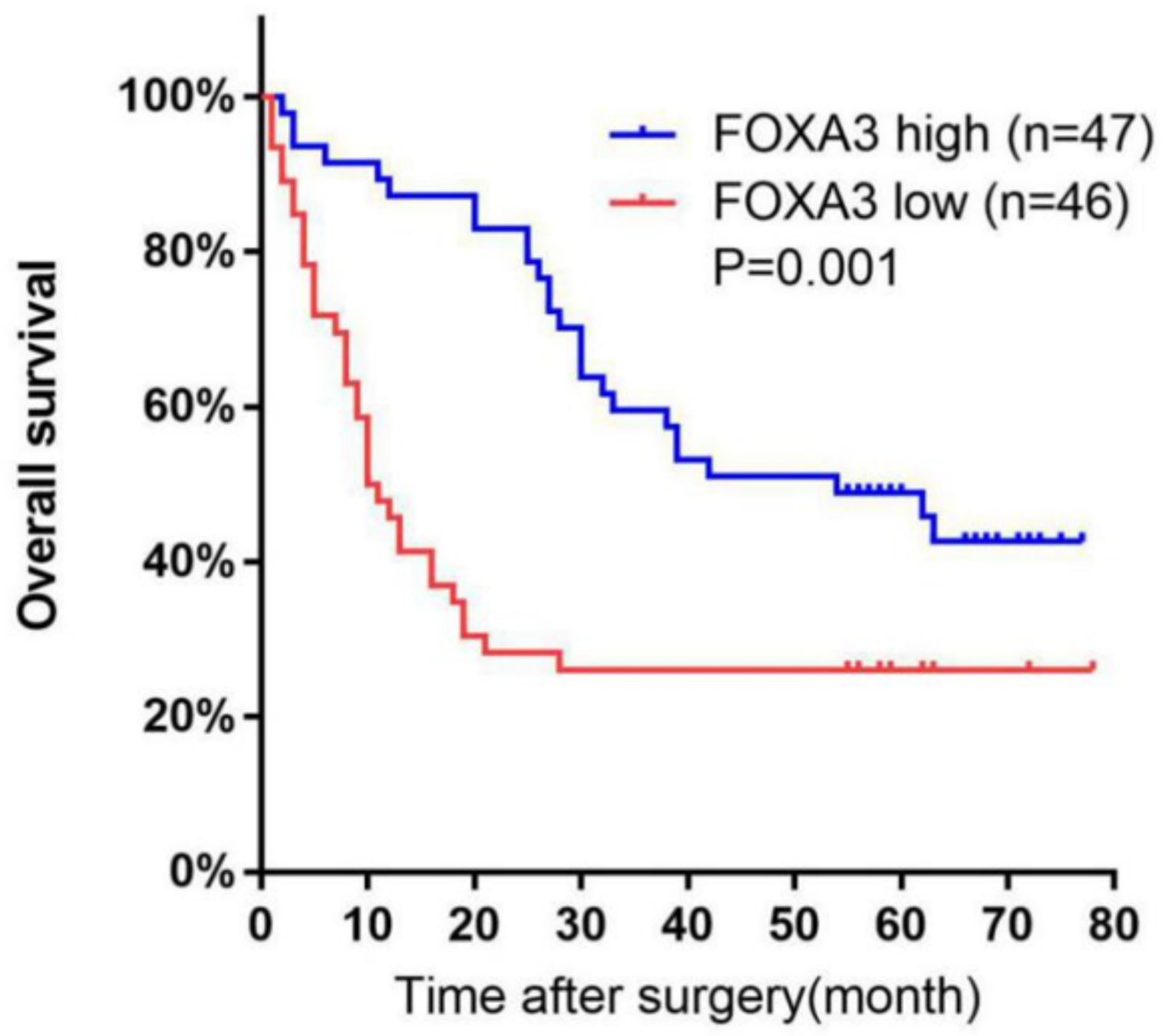

Figure 2

Estimated overall survival according to the expression of FOXA3 in 93 cases of ESCC, Kaplan-Meier survival analysis showed that ESCC patients with the low FOXA3 expression group had poorer overall survival. $(p=0.004)$ 
A

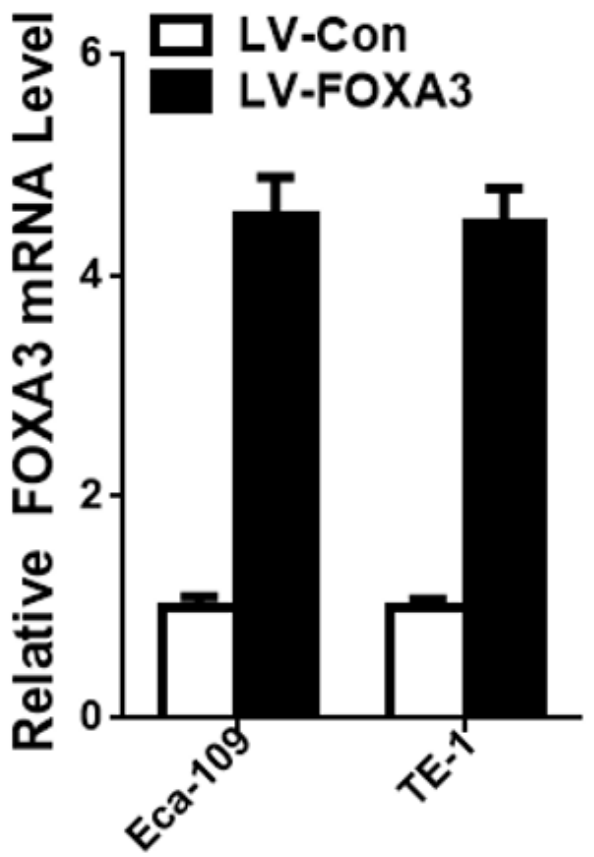

B
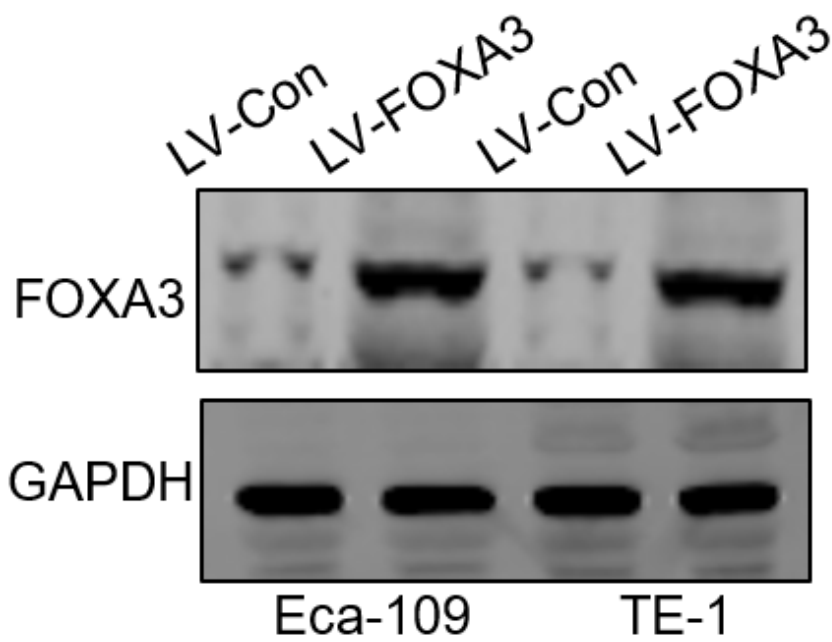

Figure 3

Generation of stable FOXA3 overexpression cell lines. A, The level of mRNA expression in LV-Con and LVFOXA3 analyzed by qRT-PCR. B, Western-blot was used to examine the expression of FOXA3. 
A
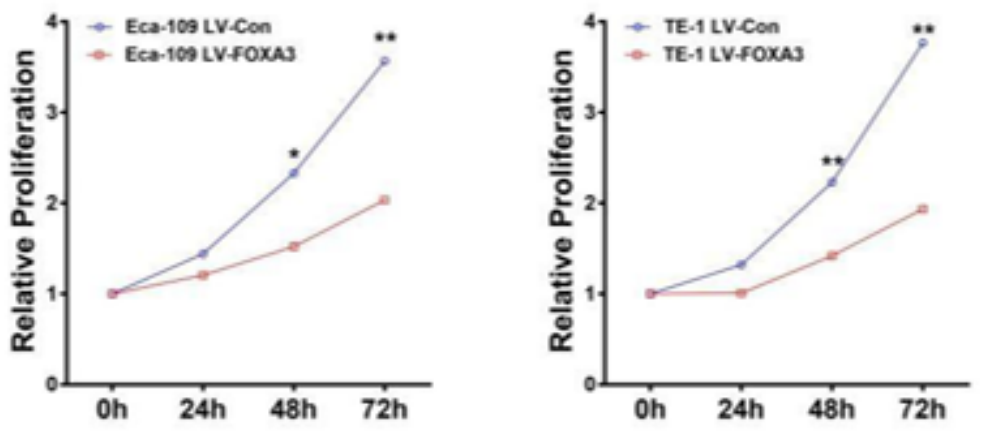

B
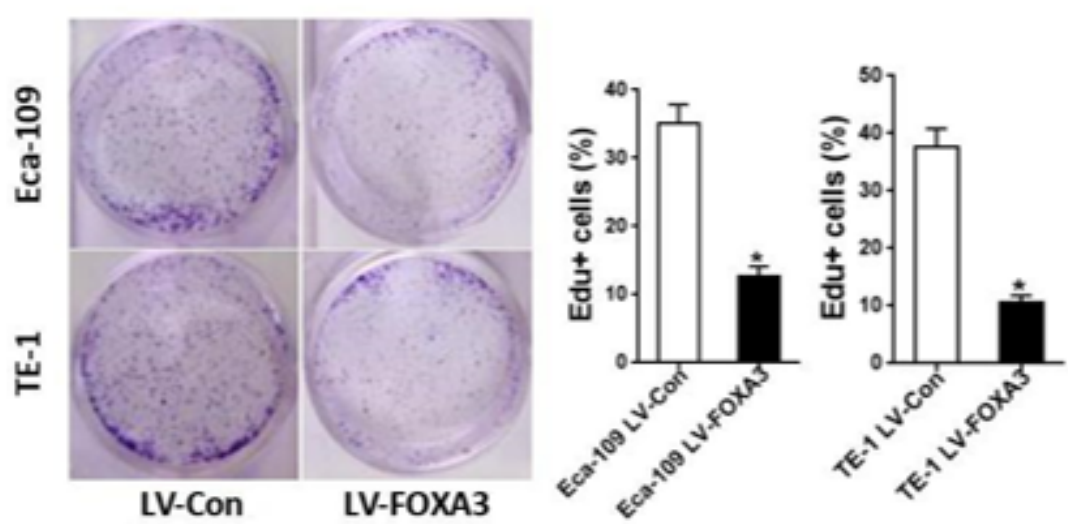

C
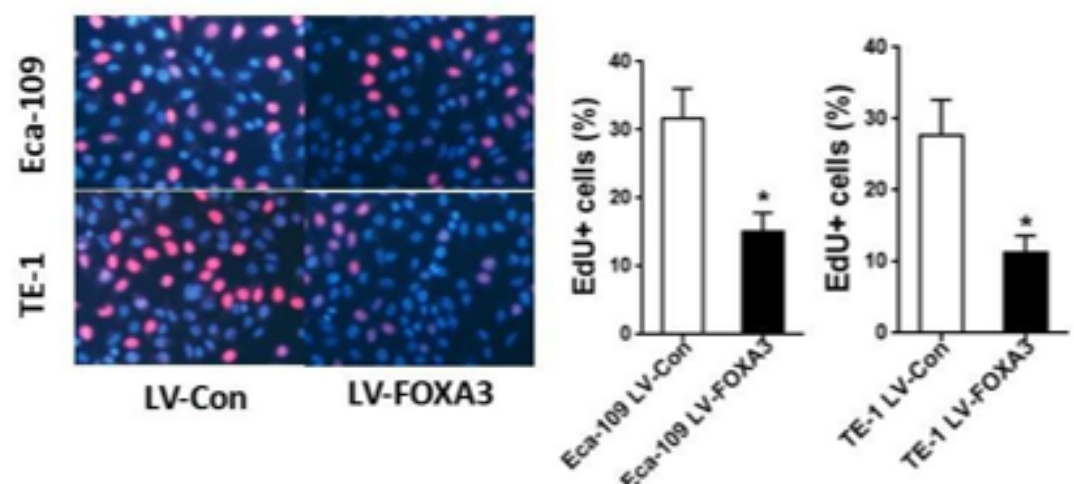

Figure 4

FOXA3 inhibits the proliferation of ESCC cells. A, Overexpression of FOXA3 inhibited the proliferation of ESCC in vitro (Eca-109, TE-1 cell lines) as compared to the control group when assessed by CCK8 assay. $B$, The suppressive effect of FOXA3 overexpression on the colony formation of ESCC cells. C, The percentage of cells binding EdU significantly decreased compared with the number in the control group. 
A $\quad$ B

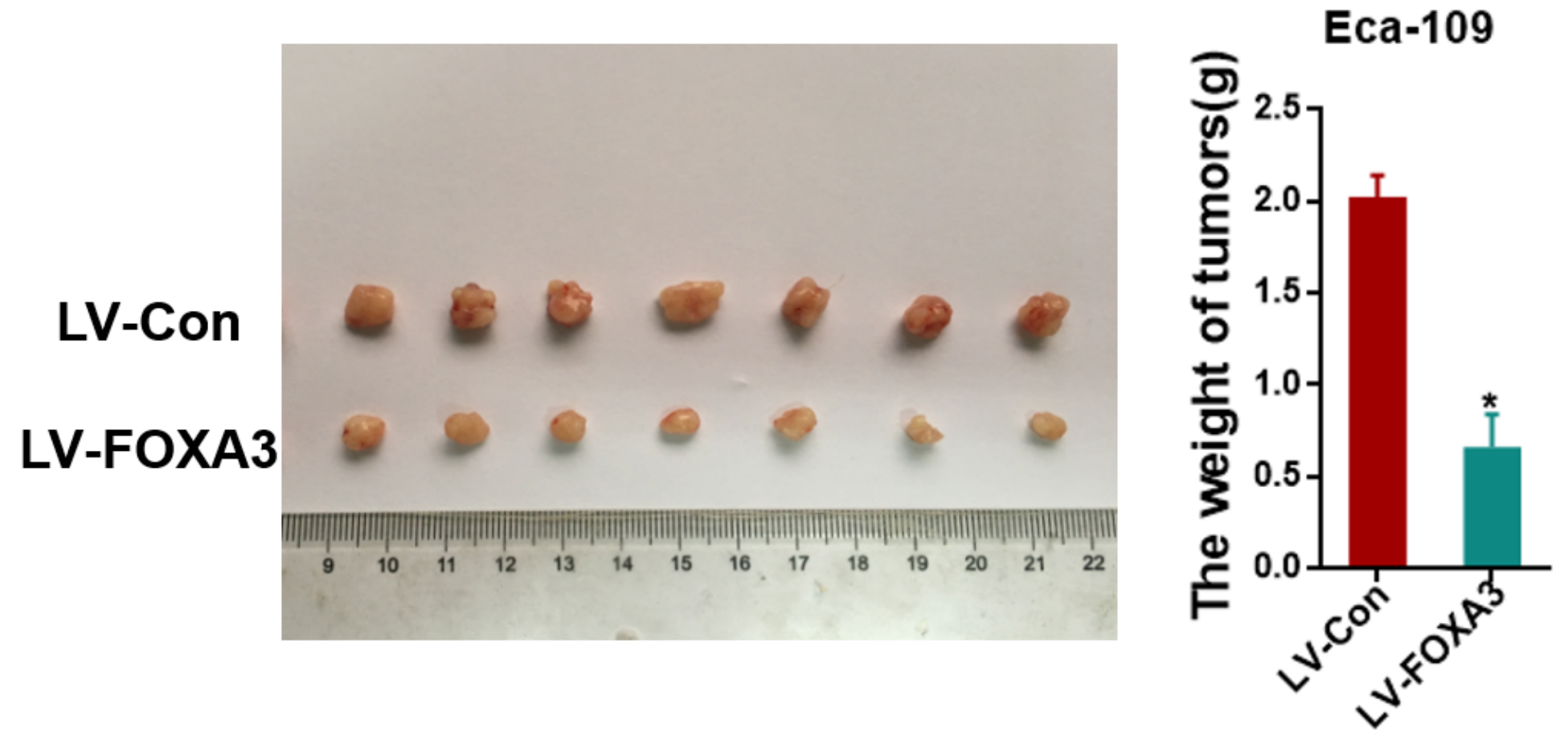

Figure 5

FOXA3 also inhibited tumor growth in vivo. A, Comparison of general view and volume of tumors between the two groups. B, Comparison of average tumor weight between the two groups. 

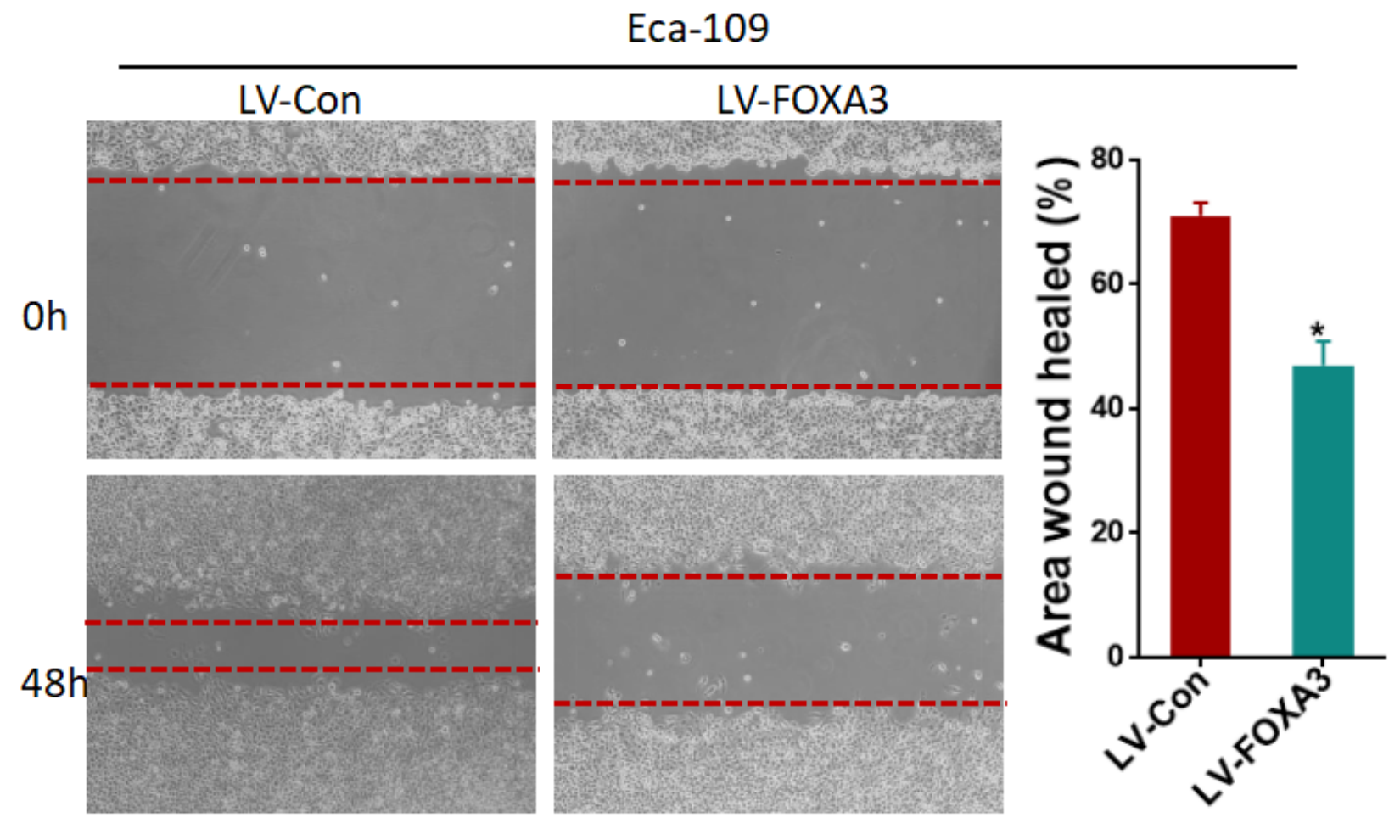

TE-1

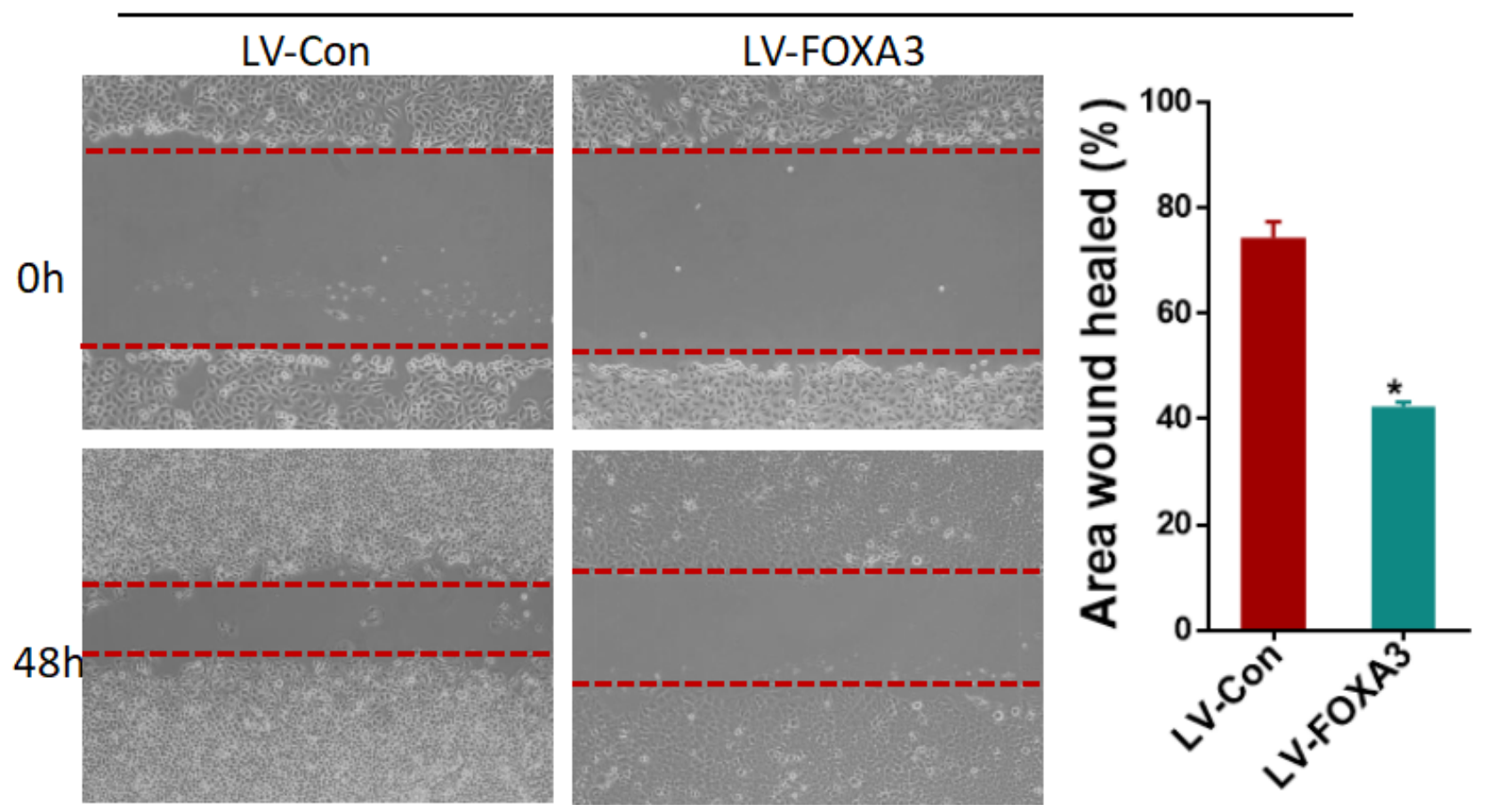

Figure 6

The migration ability of Eca-109 and TE-1 cells were evaluated by wound healing assay after overexpression of FOXA3. The migration area after $48 \mathrm{~h}$ was photographed and quantitatively analyzed. 

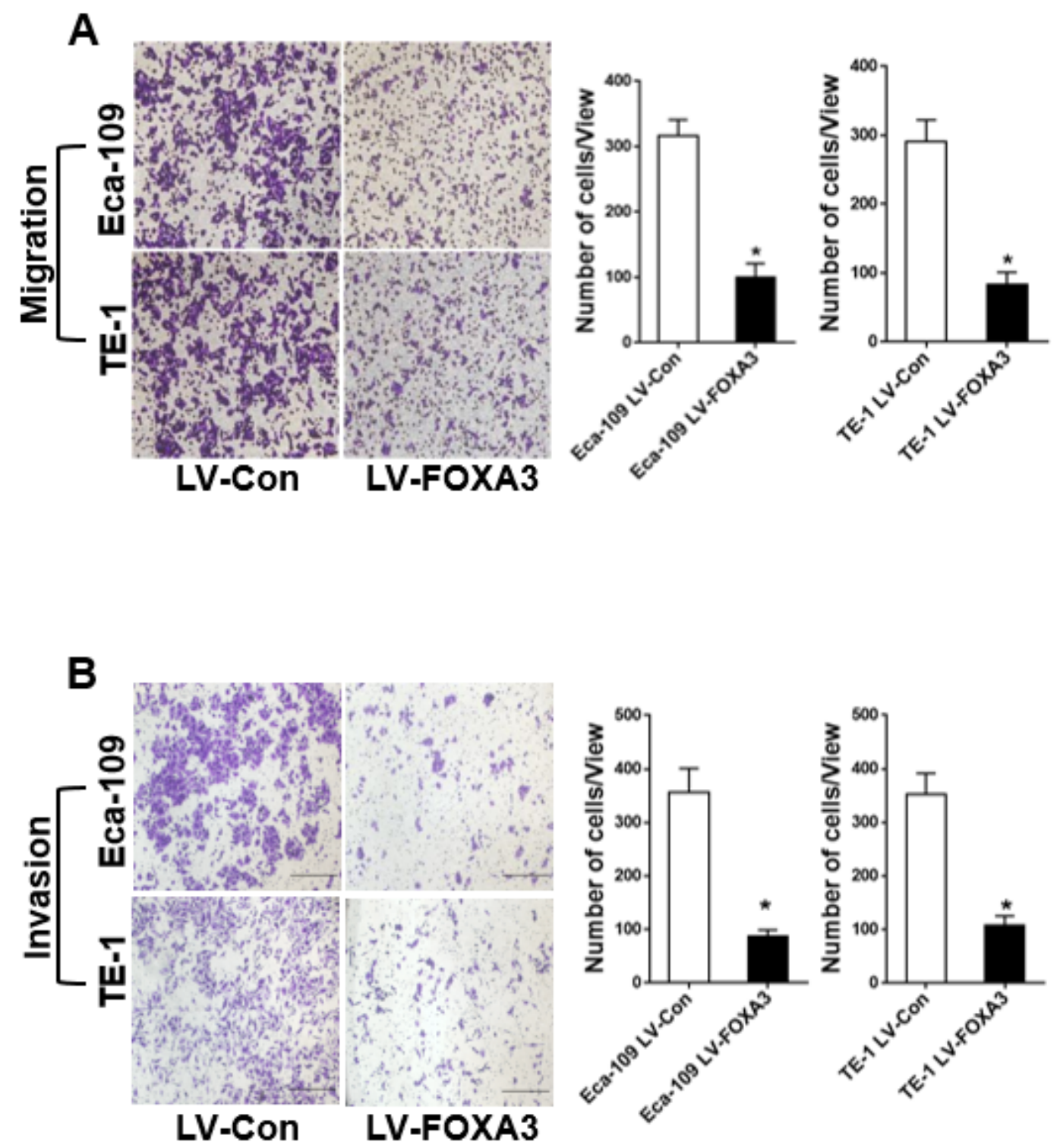

Figure 7

Transwell migration and invasion assay. A, Cells migrating in the LV-FOXA3 groups was apparently decreased compared with the control groups. B, The similar effects of FOXA3 on the invasion of Eca-109 and TE-1 cells. 